\title{
SPECTRAL PROPERTIES OF HOLOMORPHIC AUTOMORPHISM WITH FIXED POINT
}

\author{
by T. MAZUR AND M. SKWARCZYŃSKI
}

(Received 4 October, 1985)

Introduction. The Hilbert space methods in the theory of biholomorphic mappings were applied and developed by S. Bergman $[1,2]$. In this approach the central role is played by the Hilbert space $L^{2} H(D)$ consisting of all functions which are square integrable and holomorphic in a domain $D \subset \mathbb{C}^{N}$. A biholomorphic mapping $\varphi: D \rightarrow G$ induces the unitary mapping $U_{\varphi}: L^{2} H(G) \rightarrow L^{2} H(D)$ defined by the formula

$$
\left(U_{\varphi} f\right)(z)=f(\varphi(z)) \frac{\partial \varphi}{\partial z} .
$$

Here $\partial \varphi / \partial z$ denotes the complex Jacobian of $\varphi$. The mapping $U_{\varphi}$ is useful, since it permits to replace a problem for $D$ by a problem for its biholomorphic image $G$ (see for example [11], [13]). When $\varphi$ is an automorphism of $D$ we obtain a unitary operator $U_{\varphi}$ on $L^{2} H(D)$.

Since the appearance of K. Hoffman's book [7] the spaces of holomorphic functions became an interesting subject for experts in functional analysis. An important part of the research was devoted to Hardy spaces $H^{p}(D)$ and to composition operators. A holomorphic automorphism $\varphi$ of $D$ induces the composition operator $A_{\varphi}$ on $H^{p}(D)$ by the formula

$$
\left(A_{\varphi} f\right)(z)=f(\varphi(z)) .
$$

The spectral properties of (2) were studied in [6], [8], [10]. The purpose of the present paper is to establish the following spectral property of the operator (1).

THEOREM 1. Assume that $L^{2} H(D) \neq\{0\}$. Let $\varphi: D \rightarrow D$ be a holomorphic automorphism with fixed point $a \in D$. Then there is a complete orthonormal system in $L^{2} H(D)$ contained in the set of all eigenfunctions of $U_{\varphi}$.

It should be noted that, strictly speaking, $U_{\varphi}$ is not a composition operator for two reasons. First the spaces $L^{2} H(D)$ and $H^{2}(D)$ are different. (For example in the unit disc $\Delta \subset \mathbb{C}$ the first space is larger as a set than the second.) Next, in the definition (1) of $U_{\varphi}$ there is a factor $\partial \varphi / \partial z$, which does not appear in the definition (2) of $A_{\varphi}$. Our proof of Theorem 1 will be based directly on the properties of the Bergman function [2]. The assumption that $\varphi$ has a fixed point is essential. The case $D=\Delta$ was considered earlier in [9].

1. Holomorphic vector-valued functions. The following result is classical. See [12, Definition 3.30 and exercise 26].

THEOREM 2. Let $X$ be a complex Banach space. Let $D$ be a domain in $\mathbb{C}^{N}$. For every

Glasgow Math. J. 28 (1986) 25-30. 
mapping $F: D \rightarrow X$ the following conditions are equivalent

$1^{\circ}$ the function $x^{*} \circ F$ is holomorphic for every $x^{*} \in X^{*}$;

$2^{\circ}$ for every $a=\left(a_{1}, a_{2}, \ldots, a_{N}\right) \in D$ there exists a power series with coefficients in $X$ which for all $z=\left(z_{1}, z_{2}, \ldots, z_{N}\right)$ sufficiently close to a converges to

$$
F(z)=\sum_{k=\left(k_{1}, k_{2}, \ldots, k_{N}\right)} g_{k}\left(z_{1}-a_{1}\right)^{k_{1}}\left(z_{2}-a_{2}\right)^{k_{2}} \ldots\left(z_{N}-a_{N}\right)^{k_{N}} .
$$

If the above conditions are satisfied, then $F$ is called holomorphic. It is easy to verify that $F$ is holomorphic iff it is $\mathbb{C}$-differentiable. Moreover the coefficients $g_{k}$ are determined uniquely by $F$ and $a$.

2. Formal substitution and formal multiplication of power series. We shall now recall some properties of power series with coefficients in a Banach space $X$. Assume that the power series

$$
F(z)=\sum_{k=\left(k_{1}, k_{2} \ldots, k_{N}\right)} g_{k} z_{1}^{k_{1}} \cdot z_{2}^{k_{2}} \ldots z_{N}^{k_{N}}
$$

converges in an open polydisc $P=\left\{z \in \mathbb{C}^{N} ;\left|z_{j}\right|<p_{j}, j=1,2, \ldots, N\right\}$. Assume further that in an open polydisc $Q=\left\{z \in \mathbb{C}^{N} ;\left|u_{l}\right|<q_{l}, l=1,2, \ldots, N\right\}$ holomorphic functions $h_{j}$, $j=1,2, \ldots, N$ are represented by power series

$$
h_{j}(u)=\sum_{r=\left(r_{1}, r_{2}, \ldots, r_{N}\right)} c_{r}^{(j)} u_{1}^{r_{1}} u_{2}^{r_{2}} \ldots u_{N}^{r_{N}}
$$

and the mapping $h=\left(h_{1}, h_{2}, \ldots, h_{N}\right)$ satisfies $h(0)=0$. The series (4) converges absolutely and we denote

$$
\hat{h}_{j}(u)=\sum_{r=\left(r_{1}, r_{2} \ldots, r_{N}\right)}\left|c_{r}^{(j)}\right| \cdot\left|u_{1}\right|^{r_{1}}\left|u_{2}\right|^{r_{2}} \ldots\left|u_{N}\right|^{r_{N}} .
$$

A formal substitution $z=h(u)$ into the expression

$$
z_{1}^{k_{1}} \cdot z_{2}^{k_{2}} \ldots z_{N}^{k_{N}}=z^{k}
$$

requires a formal multiplication of $|k|=k_{1}+k_{2}+\ldots+k_{N}$ series which correspond to factors on the left side. This means that one has to select one term in each of these series, and then multiply the selected terms. Next one forms the sum of all products obtained in the above way.

It is now clear, how to define a formal substitution $z=h(u)$ into (3). A pedantic definition could run as follows. For a fixed $k=\left(k_{1}, k_{2}, \ldots, k_{N}\right)$ denote by $A_{k}$ the set of all sequences

$$
\sigma=\left(s_{11}, \ldots, s_{1 k_{1}}, s_{21}, \ldots, s_{2 k_{2}}, \ldots, s_{N 1}, \ldots, s_{N k_{N}}\right)
$$

of length $|k|$ with terms in $\{0,1,2, \ldots\}^{N}$. Then a formal substitution $z=h(u)$ into (5) is defined as a series $\sum_{\sigma \in A_{k}} W_{\sigma}(u)$, where

$$
W_{\sigma}(u)=\prod_{j=1}^{N} \prod_{l=1}^{k_{j}} c_{s_{i l}}^{(j)} u^{s_{i l}}
$$


A formal substitution $z=h(u)$ into (3) is defined as a series

$$
\sum_{k=\left(k_{1}, \ldots, k_{n}\right)} \sum_{\sigma \in A_{k}} g_{k} W_{\sigma}(u)
$$

With the above notation we have

THeOREm 3 [5, sec. 9.2.1]. Assume that the polydisc $Q_{0} \subset Q$ with centre 0 is so small, that

$$
\hat{h}_{j}(u)<p_{j} \quad j=1,2, \ldots, N
$$

for every $u \in Q_{0}$. Then the series (6) converges absolutely in $Q_{0}$, and its sum is $F(h(u))$. The terms in the series (6) can be reduced to yield the power series in $Q_{0}$ with the sum $F(h(u))$.

We shall also need the following (obvious)

THEOREM 4. Assume that both series

$$
c=\sum_{m} c_{m} \quad c_{m} \in \mathbb{C}, \quad v=\sum_{k} v_{k} \quad v_{k} \in X
$$

converge absolutely. Then the series $\sum_{k, m} c_{m} v_{k}$ converges absolutely and its sum is $c v$.

3. Subspaces generated by coefficients. For every $z \in D$ the evaluation functional

$$
L^{2} H(D) \ni f \stackrel{\mathscr{x}_{z}^{*}}{\longrightarrow} f(z) \in \mathbb{C}
$$

belongs to $L^{2} H(D)^{*}$, the space of all continuous linear functionals on $L^{2} H(D)$. (The element in $L^{2} H(D)$ which represents $\mathscr{X}_{z}^{*}$ in terms of the scalar product is denoted by $\mathscr{X}_{z}$, and $K_{D}(w, z)=\left\langle\mathscr{X}_{z}, \mathscr{X}_{w}\right\rangle w, z \in D$ is the Bergman function of $D$.)

LEMMA 1. The mapping

$$
D \ni z \mapsto \mathscr{X}_{z}^{*} \in L^{2} H(D)^{*}
$$

is holomorphic.

Proof. Set $X=L^{2} H(D)^{*}$. Since $L^{2} H(D)$ is reflexive we have $X^{*}=L^{2} H(D)$. Now, for every $f \in L^{2} H(D)$

$$
f\left(\mathscr{C}_{z}^{*}\right)=\mathscr{X}_{z}^{*}(f)=f(z)
$$

is holomorphic as function of $z$. Therefore the mapping (7) satisfies condition $1^{\circ}$ of Theorem 2, Q.E.D.

Assume now that $\varphi: D \rightarrow D$ is a holomorphic automorphism. The well-known transformation rule for the Bergman function

$$
K_{D}(w, z)=K_{D}(\varphi(w), \varphi(z))\left(\frac{\partial \varphi}{\partial w}\right)\left(\frac{\partial \varphi}{\partial z}\right)
$$

can be written as

$$
\mathscr{X}_{z}=\left(U_{\varphi} \mathscr{X}_{\varphi(z)}\right)\left(\overline{\frac{\partial \varphi}{\partial z}}\right)
$$


To derive an analogous formula for $\mathscr{Q}_{z}^{*}$ we introduce the operator $U_{\phi}^{*}: L^{2} H(D)^{*} \rightarrow$ $L^{2} H(D)^{*}$ adjoint to $U_{\varphi}$. By definition, for every $g^{*} \in L^{2} H(D)^{*}$ and every $f \in L^{2} H(D)$

$$
\left(U_{\varphi}^{*} g^{*}\right)(f)=g^{*}\left(U_{\varphi} f\right) \text {. }
$$

For $g^{*}=\mathscr{X}_{z}^{*}(8)$ yields

$$
\left(U_{\varphi}^{*} \mathscr{X _ { z } ^ { * }}\right)(f)=\mathscr{X}_{z}^{*}\left(U_{\varphi} f\right)=f(\varphi(z)) \frac{\partial \varphi}{\partial z}=\frac{\partial \varphi}{\partial z} \mathscr{X}_{\varphi(z)}^{*}(f) .
$$

Since $f \in L^{2} H(D)$ is arbitrary, we can write simply

$$
U_{\varphi}^{*} \mathscr{X}_{z}^{*}=\frac{\partial \varphi}{\partial z} \mathscr{X}_{\varphi(z)}^{*}
$$

Let us now make additional assumption that $0 \in D$ is a fixed point of $\varphi$. By Theorem 2 the functional $\mathscr{Q}_{z}^{*}$ is represented for $z$ close to 0 by the series

$$
\mathscr{L}_{z}^{*}=\sum_{k=\left(k_{1}, \ldots, k_{N}\right)} g_{k}^{*} z^{k}
$$

We can substitute (10) into the left side of (9). For $z$ close to 0 the value $\varphi(z)$ is also close to 0 , and (9) yields

$$
\sum_{k=\left(k_{1}, \ldots, k_{N}\right)}\left(U_{\varphi}^{*} g_{k}^{*}\right) z^{k}=\frac{\partial \varphi}{\partial z} \sum_{k=\left(k_{1}, \ldots, k_{N}\right)} g_{k}^{*}(\varphi(z))^{k}
$$

We shall now express the right side of (11) as power series in the variable $z$; since the coefficients in such expansion are uniquely determined this will yield information about $U_{p}^{*} g_{k}^{*}$. To this end we develop $\partial \varphi / \partial z$ as power series about 0 , and compute formally the right side of (11) using Theorem 3 and Theorem 4. The resulting series converges absolutely for $z$ close to 0 , and, after reduction yields the desired power series.

Denote $\varphi=\left(\varphi_{1}, \varphi_{2}, \ldots, \varphi_{N}\right)$. We are now ready for the following crucial observation. Since $\varphi(0)=0$, the development of $\varphi_{j}, j=1,2, \ldots, N$ has no zero order terms. Therefore for every $s=0,1,2, \ldots$ all terms in the development of

$$
\varphi(z)^{k}=\varphi_{1}(z)^{k_{1}} \varphi_{2}(z)^{k_{2}} \ldots \varphi_{N}(z)^{k_{N}}, \quad|k|>s
$$

have order greater than $s$. Therefore for every multiindex $m=\left(m_{1}, m_{2}, \ldots, m_{N}\right)$ such that $|m| \leqslant s$ the coefficient $U_{\varphi}^{*} g_{m}^{*}$ is equal to the coefficient at $z^{m}$ in the expression

$$
\frac{\partial \varphi}{\partial z} \sum_{|k| \leqslant s} g_{k}^{*}(\varphi(z))^{k}
$$

It follows that $U_{q}^{*} g_{m}^{*},|m| \leqslant s$ belongs to the subspace $H_{s}^{*}$ in $L^{2} H(D)^{*}$ generated by elements $g_{k}^{*},|k| \leqslant s$. We can now state

Lemma 2. For every $s=0,1,2, \ldots$

$$
U_{\varphi}^{*} H_{s}^{*}=H_{s}^{*}
$$


Proof. We have seen already that $U_{p}^{*} H_{s}^{*} \subset H_{s}^{*}$. Now $U_{\varphi}^{*}$ is a linear automorphism of $L^{2} H(D)^{*}$ (since $U_{\varphi}$ is a linear automorphism of $L^{2} H(D)$ ). Therefore $\operatorname{dim} U_{\varphi}^{*} H_{s}^{*}$ is equal to the (finite) number $\operatorname{dim} H_{s}^{*}$. It follows that the inclusion is in fact an equality, Q.E.D.

Denote by $\pi$ the mapping of $g^{*} \in L^{2} H(D)^{*}$ onto the unique element $g \in L^{2} H(D)$ which represents $g^{*}$ in terms of the scalar product. It is well known that $\pi$ is conjugate linear and norm-preserving. It maps $H_{s}^{*}$ onto the subspace $H_{s}$ in $L^{2} H(D)$ generated by elements $g_{k}=\pi\left(g_{k}^{*}\right),|k| \leqslant s$. We can restate Lemma 2 as follows.

Lemma 3. For every $s=0,1,2, \ldots$

$$
U_{\varphi} H_{s}=H_{s}
$$

Proof. Set $U=\pi U_{\varphi^{-1}}^{*} \pi^{-1}$. Lemma $2\left(\right.$ for $\left.\varphi^{-1}\right)$ implies that

For every $g, f \in L^{2} H(D)$

$$
U H_{s}=H_{s} \text {. }
$$

$$
\begin{aligned}
\langle U g, f\rangle & =\left\langle\overline{f, \pi U_{\varphi^{-1}}^{*} \pi^{-1} g}\right\rangle=\left(\overline{\left.U_{\varphi^{-1}}^{*} \pi^{-1} g\right)(f)}=\overline{\left(\overline{\left.\pi^{-1} g\right)\left(U_{\varphi^{-1}} f\right.}\right)}\right. \\
& =\left\langle\overline{U_{\varphi^{-1}} f, g}\right\rangle=\left\langle\overline{f, U_{\varphi} g}\right\rangle=\left\langle U_{\varphi} g, f\right\rangle
\end{aligned}
$$

(we used the fact that $U_{\varphi}$ is unitary, and $U_{\varphi}^{-1}=U_{\varphi^{-1}}$. It follows that $U=U_{\varphi}$ and (14) implies (13), Q.E.D.

Denote by $\hat{H}_{s}, s=1,2, \ldots$ the orthogonal complement of $H_{s-1}$ in $H_{s}$. Since $U_{q}$ preserves scalar product, from Lemma 3 follows

Lemma 4. For every $s=1,2, \ldots$

$$
U_{\varphi} \hat{H}_{s}=\hat{H}_{s}
$$

4. Proof of Theorem 1. We can assume (with no loss of generality) that $0 \in D$ is a fixed point for $\varphi$. Define

$$
E_{0}= \begin{cases}\left\{g_{0} /\left\|g_{0}\right\|\right\} & \text { if } g_{0} \neq 0 \\ \varnothing & \text { otherwise }\end{cases}
$$

We shall define $E_{s}, s=1,2, \ldots$ as follows. If $\hat{H}_{s} \neq\{0\}$ we take for $E_{s}$ any othonormal system of eigenfunctions for $U_{p}: \hat{H}_{s} \rightarrow \hat{H}_{s}$ which generates $\hat{H}_{s}$. (Such system exists by spectral theorem for unitary operator in a finite dimensional space.) If $\hat{H}_{s}=\{0\}$ we set $E_{s}=\varnothing$. Denote $E=\bigcup_{s=0}^{\infty} E_{s}$. By construction, every non zero coefficient $g_{k}$ can be written as linear combination of elements in $E$. Note that the set of all $g_{k}$ is total in $L^{2} H(D)$ because $g_{k}$ (up to a positive constant) represents the partial derivative (a $\partial^{|k|}$ $\left.\partial z_{1}^{k_{1}} \ldots \partial z_{N}^{k_{N}}\right)\left.\right|_{z=0}$. If $E=\varnothing$ then $L^{2} H(D)=\{0\}$, contrary to the assumption. Hence $E \neq \varnothing$ is (by construction) an orthonormal system in $L^{2} H(D)$. This system is complete, since it spans a total set of all $g_{k}$, Q.E.D.

5. An example: complete circular domains. A domain $D \Subset \mathbb{C}^{N}$ is called complete circular (with respect to $0 \in \mathbb{C}^{N}$ ) if for every $z \in D$, and every $\lambda \in \mathbb{C}$ such that $|\lambda| \leqslant 1$, the 
point $\lambda z=\left(\lambda z_{1}, \ldots, \lambda z_{N}\right)$ belongs to $D$. For such domains (and $\left.a=0\right)$ Theorem 1 can be obtained directly from the following result of H. Cartan [3], [4].

Theorem 5. Let $D \Subset \mathbb{C}^{N}$ be a complete circular domain. Then

(a) every holomorphic automorphism $\varphi: D \rightarrow D$ such that $\varphi(0)=0$ can be written as $\varphi=\left(\varphi_{1}, \varphi_{2}, \ldots, \varphi_{N}\right)$ where

$$
\varphi_{i}(z)=a_{i 1} z_{1}+\ldots+a_{i N} z_{N}
$$

and $a_{i j} \in \mathbb{C}$ for $i, j=1,2, \ldots, N$,

(b) homogeneous polynomials of different degrees are orthogonal in $L^{2} H(D)$,

(c) the set of all polynomials is dense in $L^{2} H(D)$.

Indeed, (a) implies that $\partial \varphi / \partial z \equiv$ const, and $U_{\varphi}$ maps the (finite dimensional) subspace of all homogeneous polynomials of degree $s$ onto itself. Using (b) we can construct an orthonormal system of eigenfunctions for $U_{\varphi}$ which spans every polynomial. This system is complete by (c). This proves Theorem 1 in the considered case. Perhaps the interest in Theorem 1 is justified by its relation with Theorem 5 .

\section{REFERENCES}

1. S. Bergman, Über die Kernfunktion eines Bereiches und ihr Verhalten am Rande, J. Reine Angew. Math. 169 (1933), 1-42 and 172 (1934), 89-123.

2. S. Bergman, The kernel function and conformal mapping, Math. Surveys 5, second ed. (Amer. Math. Soc., 1970).

3. H. Cartan, Les fonctions de deux variables complexes et le problème de la representation analytique, J. Math. Pures Appl. (9) 10 (1931), 1-114.

4. H. Cartan, Sur les groupes de transformations analytiques, Actualités Sci. Indust., Exposés Math. 9 (Paris, 1935).

5. J. Dieudonné, Foundations of modern analysis (Academic Press, 1960).

6. S. Fisher, Eigenvalues and eigenvectors of compact composition operators on $H^{p}(\Omega)$, Indiana Univ. Math. J. 32 (1983), 843-847.

7. K. Hoffman, Banach spaces of analytic functions (Prentice-Hall, 1962). $132-150$.

8. H. Kamowitz, The spectra of composition operators on $H^{p}, J$. Funct. Anal. 18 (1975),

9. T. Mazur, Spectral properties of automorphisms of the unit disc, Demonstratio Math., 17 (1984), 1069-1072.

10. A. Nordgren, Composition operators on Hilbert space, in J. M. Bachar Jr. and D. W. Hadwin, Hilbert space operators, proceedings, 1977, Lecture Notes in Mathematics 693 (Springer, 1978), 37-63.

11. I. Ramadanov and $M$. Skwarczyński, An angle in $L^{2}(\mathbb{C})$ determined by two plane domains, Bull. Acad. Polon. Sci., to appear.

12. W. Rudin, Functional Analysis (McGraw-Hill, 1973).

13. M. Skwarczyński, The invariant distance in the theory of pseudo-conformal transformations and the Lu Qi-keng conjecture, Proc. Amer. Math. Soc. 22 (1969), 305-310.

MaCiej SKWARCZYŃSKI

01698 WARSAW

SMOLENSKIEGo $27 \mathrm{a}$ m. 14

POLAND 